\title{
Envelhecimento sadio, Comprometimento Cognitivo Leve e doença de Alzheimer: um estudo das estratégias comunicativas na narrativa oral
}

\author{
Healthy aging, Mild Cognitive Impairment and Alzheimer's disease: \\ a study of the communicative strategies in oral narratives
}

Gislaine Machado Jerônimo

Pontifícia Universidade Católica do Rio Grande do Sul, Porto Alegre, RS, Brasil

$\diamond$

\begin{abstract}
Resumo: O aumento de casos de Comprometimento Cognitivo Leve (CCL) e de doença de Alzheimer (DA) leva ao questionamento sobre as habilidades cognitivas e linguísticas comprometidas. A literatura, de modo geral, aborda os prejuízos na comunicação destas populações, sendo pouco investigadas as habilidades preservadas. Assim, este estudo objetiva analisar as estratégias comunicativas (ECs) presentes na produção de narrativa oral desses indivíduos, bem como verificar o impacto da variável escolaridade no desempenho da mesma atividade em idosos saudáveis. Participaram do estudo 95 idosos, os quais produziram uma narrativa oral baseada em uma sequência de figuras, The Dog Story (LE BOUEF, 1976). Os resultados mostraram que idosos com CCL e DA fizeram mais uso de ECs do que o grupo controle, ao passo que idosos saudáveis com alta escolaridade utilizaram menos. Portanto, as ECs mostraram-se um apoio à comunicação no CCL e na DA, assim como a escolaridade evidenciou-se um importante marcador de reserva cognitiva no envelhecimento sadio.
\end{abstract}

Palavras-chave: Estratégias comunicativas; Narrativa; Envelhecimento

\begin{abstract}
The increase in cases of the Mild Cognitive Impairment (CCL) and Alzheimer's disease (AD) requires more studies on impaired cognitive and linguistic abilities. Literature, in general, addresses the communication losses of these populations, but with little attention to the investigation of preserved skills. Thus, this study aims at analyzing the communicative strategies (CSs) present in the oral narrative production of these individuals, as well as to verify the impact of the educational variable on the performance of the same activity in healthy elderly people. The study involved 95 elderly people, who produced an oral narrative based on a sequence of figures, The Dog Story (LE BOUEF, 1976). The results showed that the elderly with CCL and AD had higher use of CSs than the control group, while healthy elderly with high schooling used fewer CSs. Therefore, the analyses of CSs showed to be an supporting aid for the communication in CCL and $\mathrm{AD}$, as well as schooling was evidenced as an important marker of cognitive reserve in healthy aging.
\end{abstract}

Keywords: Communicative strategies; Narrative; Aging

\section{Introdução}

A população idosa brasileira está em constante crescimento, o que amplia o quadro de doenças relacionadas à idade como é o caso do Comprometimento Cognitivo Leve (CCL) e da doença de Alzheimer (DA) (GOMES; TERRA, 2015; TOLEDO et al., 2017). O CCL configura-se como um declínio cognitivo associado à idade, um estágio de transição entre o envelhecimento sadio e um processo demencial. Para que se caracterize como um quadro de CCL, observam-se os seguintes critérios: a) queixa por parte do idoso ou de algum familiar, ou por parte médica; b) alteração em um ou mais domínios da cognição e c) preservação funcional, isto é, o idoso precisa manter autonomia nas atividades de vida diária. Quando os domínios cognitivos afetam a memória, o CCL é chamado de CCL amnéstico, sendo este o tipo de CCL que pode vir a tornar-se DA (ALBERT et al., 2011). 
Já a DA caracteriza-se como uma doença neurodegenerativa progressiva. Tanto no CCL como na DA precisa haver manifestação de queixa. No entanto, para configurar-se como um quadro de DA é necessário haver prejuízos em no mínimo dois domínios da cognição, bem como comprometimento na funcionalidade, isto é, o paciente perde sua autonomia nas atividades de vida diária (MCKHANN et al., 2011).

No envelhecimento saudável, mais do que a presença ou ausência de doença está a manutenção da habilidade funcional (OMS, 2015). A alta escolaridade, neste caso, pode ser um importante recurso, o qual vem ao encontro da Hipótese de Reserva Cognitiva (HRC). A HRC trata dos fatores que podem interferir positivamente para atenuar o declínio cognitivo típico do envelhecimento. Esta reserva seria uma economia de recursos a serem utilizados de acordo com a demanda e pode ser adquirida por meio da leitura, aprendizagem de outros idiomas, alta escolaridade, a profissão com demanda cognitiva, dentre outros fatores.

O CCL e a DA têm imposto certos desafios à comunidade científica, uma vez que o CCL pode tornar-se DA, e na DA há um declínio progressivo das capacidades intelectuais, acometendo a memória e também outros componentes, dentre eles, a linguagem. Neste aspecto, tem-se pouco conhecimento sobre as características da produção oral no CCL e na DA,sendo a narrativa uma fonte rica de evidências de tais prejuízos, bem como evidência dos possíveis recursos remanescentes (JERÔNIMO; HÜBNER, 2014).

No campo da Psicolinguística, Neurolinguística e Neuropsicologia as falhas no processamento do discurso são investigadas, especialmente quando as populações estudadas apresentam DA ou algum outro tipo de lesão cerebral (CARDEBAT; DÉMONET; DOYON, 1993, DOUNG et al., 2003; BRANDÃO, 2005). Visando a superar as dificuldades encontradas, o sujeito pode utilizar-se de estratégias de comunicação, dentre elas hesitações, paráfrases, neologismos, correções, repetições, parafasias e circunlóquios.

Este estudo tem por objetivoanalisar as estratégias comunicativas (ECs) presentes na produção de narrativa oral de idosos com CCL e DA, bem como aprofundar o conhecimento sobre o impacto da variável escolaridade na produção de narrativa oral de idosos saudáveis.

\section{Breve revisão das estratégias comunicativas na narrativa}

A produção narrativa não envolve somente $\mathrm{o}$ planejamento, mas igualmente sua realização. Ao produzir uma narrativa oral, o locutor frequentemente está em relação com um interlocutor, que tem por objetivo compreendê-la. Assim, o locutor deve produzir uma narrativa que seja compreensível. Entretanto, falhas no processo podem ocorrer, pois o locutor pode encontrar problemas na sua formulação, precisando resolvê-las (FAVERO et al., 2000).

A iniciativa de resolução de problemas, no que tange à formulação oral, está associada com a capacidade de adequação pragmática do sujeito, envolvendo um esforço cognitivo para dar conta das falhas encontradas. Tal adequação pragmática está relacionada com o componente de K-Device - proposto por Van Dijk (2003), o qual tem por objetivo adequar a fala do sujeito ao contexto.

ECs são consideradas, ao invés de manifestações negativas, importantes estratégias cognitivas e de comunicação, utilizadas especialmente quando há a falta de uma informação ou de uma expressão adequada. Elas são valiosa fonte de informação para um estudo qualitativo do processamento pragmático necessário para a realização discursiva (WEIRATHER, 2010).

Hesitações são estratégias utilizadas especialmente na ocorrência de quebra na linearidade, ou seja, na formulação da ideia a ser enunciada. Trata-se de uma dificuldade relativa ao tópico, a qual pode ser resolvida através de pausas curtas ou mais longas, alongamento das palavras e até risos. Fazendo uso destes recursos, ganha-se tempo até encontrar a palavra ou termo alvo (FÁVERO et al., 2000). No entanto, este tempo extra pode causar uma quebra no tópico, deixando o locutor com dificuldade de recuperá-lo.

A paráfrase é entendida como uma atividade de reformulação de um enunciado anterior, mantendo uma relação de equivalência. Por exemplo: o carro está muito velho... carro muito usado... o carro não é novo (FÁVERO et al., 2000). Outro entendimento de paráfrase é a tentativa de uma enunciação que, devido à anomia, por exemplo, não ocorre; a palavra é substituída por outra expressão ou frase que a explique. Seria o caso de, por exemplo, para caneta, dizer "aquilo com que se que escreve" (ORTIZ, 2010, p. 73).

Repetição é outra característica discursiva, a qual pode ser utilizada na narração, que por muito tempo foi vista como um aspecto negativo. No entanto, atualmente sabe-se que a repetição pode ser um importante elemento retórico, que traz efeitos semânticos de persuasão no interlocutor, sendo igualmente um recurso coesivo de referenciação e de coesão sequencial. Trata-se de um recurso que sinaliza categorias, diferenciando o que é novo do que já é conhecido. Enquanto estratégia de comunicação, a repetição é utilizada para compensar dificuldades encontradas (TOMOEDA; BAYLES, 1993). A repetição compreende aquelas enunciações produzidas pelo próprio falante com o objetivo de ganhar tempo para o planejamento ou recordação da palavra. Esse tempo assegura a manutenção do turno, no caso de uma conversação $(\mathrm{KOCH}, 2008)$. A dificuldade de achar 
palavras também pode vir acompanhada do fenômeno chamado de "Fenômeno da Ponta da Língua", do inglês "Tip of the Tongue", em que a pessoa parece ter consciência da palavra que quer enunciar, mas não consegue recuperála da memória (SCHWARTZ; METCALFE, 2011; CAIXETA et al., 2014).Nestes casos, pode-se repetir a última palavra enunciada à espera da palavra seguinte, aquela que completa a expressão ou ideia. A repetição, assim, pode funcionar como pista para encontrar a palavra alvo. Por exemplo, "a cidade de ...de...de".

A parafasia, nomenclatura que vem do campo da Afasiologia, trata da troca de uma palavra, considerada a palavra-alvo, por outra palavra semântica ou fonologicamente relacionada (TUBERO, 2010). Desse modo, as parafasias se dividem em vários subtipos, entre os mais comuns estão a parafasia semântica e a parafasia fonética ou fonêmica. No primeiro subtipo, há uma troca da palavra-alvo por outra palavra do mesmo campo semântico. Por exemplo, falar "faca", ao invés de "garfo". No segundo subtipo, há ocorrência de omissão, adição, duplicação e substituição de letras. Exemplo, "gatei", por "gastei", "dilzer" por "dizer", "cachorrorroo" por "cachorro" e "fapato" por "sapato" (JAKUBOVICZ; CUPELLO, 2005).

É importante ressaltar que as parafasias nem sempre são processos conscientes e usados como estratégias, pois elas podem aparecer na fala involuntariamente. Posterior a uma parafasia involuntária, por exemplo, a fim de reaver o sentido desejado, é comum a autocorreção da palavra, sendo essa outra estratégia de comunicação. Segundo Fávero e colegas (2000), a correção ou autocorreção também é frequente quando a pessoa muda o planejamento ao longo da construção do texto oral, fazendo-se necessária para situar o interlocutor dos novos caminhos estabelecidos.

Neologismos igualmente podem aparecer, são bastante recorrentes nos casos em que a pessoa não lembra a palavra-alvo, mas tenta enunciar algo parecido. No caso do neologismo, a pessoa acaba, nessa aproximação, criando uma palavra inexistente na língua. "Por exemplo, eu fui pegar um necape", ao invés de "Nescafé" (ORTIZ, 2010, p. 75).

Por fim, os circunlóquios podem aparecer quando há uma evidente dificuldade de discorrer sobre o tópico escolhido. Desse modo, a pessoa tangencia o tema, não conseguindo falar sobre ele. Há uma tentativa de estabelecer um contato com o interlocutor, porém essa tentativa é frustrada em termos de conteúdo, pois claramente o locutor demonstra dificuldade de acesso ao léxico. Entretanto, poderia se dizer que, nesse caso, além da dificuldade salientada, há um comprometimento no planejamento do tópico, sendo que um prejuízo pode ser decorrente do outro.
As estratégias comunicativas na produção narrativa estão relacionadas à adequação pragmática da situação. Entretanto, é importante ressaltar que tais estratégias se relacionam a dificuldades de acesso ao léxico, bem como a dificuldades em outros componentes cognitivos, sejam eles os diferentes tipos de memória, a atenção e as funções executivas. Isto quer dizer que indivíduos que têm melhores condições em tais componentes, tendem a manter o tópico narrativo/discursivo e utilizar menos as estratégias mencionadas.

\section{Delineamento metodológico}

Estudo transversal, observacional e contemporâneo realizado com aprovação do Comitê de Ética em Pesquisa da PUCRS, sob o número de parecer 560.073, no dia 06 de março de 2014, registro CAAE 21006913.0.0000.5336.

\section{Participantes}

Os participantes foram selecionados através de uma amostra por conveniência,divididos em quatro grupos: idosos saudáveis com baixa escolaridade, idosos saudáveis com alta escolaridade e dois grupos clínicos diagnosticados, respectivamente, com CCL e provável DA. Foram pareados por idade(entre 60 e 79 anos), escolaridade (entre 2 e 8 anos de ensino formal para grupo de baixa escolaridade; e entre 12 e 18 anos para grupo de alta escolaridade) e quanto ao grau de demência e gravidade da doença, no caso do grupo com DA e CCL.

Ambos os grupos clínicos possuem baixa escolaridade. O grupo de idosos saudáveis com baixa escolaridade constitui o grupo controle para os grupos com CCL e DA, bem como grupo comparativo para os idosos com alta escolaridade. O diagnóstico de CCL e DA foi realizado por uma equipe multidisciplinar de um hospital em Porto Alegre, composta por neurologistas, psiquiatras, psicólogo e fonoaudiólogo, conforme recomendações do Grupo de Trabalho do National Institute on Aging (NIA) e Alzheimer's Association (AA) (ALBERT et al., 2011), através dos seguintes testes: Mini Exame do Estado Mental (MEEM), Escala de Depressão Geriátrica (GDS) e Clinical Dementia Rating (CDR) - Avaliação clínica de demência.

Todos os participantes manifestaram concordância em participar do estudo e assinaram o Termo de Consentimento Livre e Esclarecido (TCLE). Critérios de exclusão: idosos com história de alcoolismo, de doença neurológica (exceto CCL e DA), depressão, problemas relacionados à visão ou à audição não corrigidos e escolaridade abaixo de 2 anos de ensino formal; no caso de participantes com DA, CDR superior a 1; e para os idosos com CCL, CDR superior a 0,5 e ser diagnosticado com CCL de outros tipos, que não o amnéstico. 


\section{Instrumento linguístico}

A análise foi realizada com base em uma produção de narrativa oral, através de uma sequência de sete figuras em preto e branco, História do Cachorrinho (LE BOUEF, 1976). Os participantes do estudo foram individualmente convidados a contar uma história com base na sequência de figuras abaixo, diâmetro de $8 \mathrm{~cm} \times 8 \mathrm{~cm}$ (Figura 1). As cenas foram apresentadas em linha, todas ao mesmo tempo, na ordem da história.

A instrução dada é a seguinte: "vou lhe mostrar uma história com figuras. Cada figura é um momento da história, que tem início, meio e fim. Vou pedir que o(a) senhor (a) olhe bem as figuras e tente entender a história. Então, vou lhe pedir que me conte essa história como se fosse contar para um amigo/amiga. (Esperar) Está pronto(a)? Podemos começar?"

Em um primeiro momento, o participante visualiza as cenas e só começa a contar a história quando sinaliza que entendeu a instrução e está pronto para começar. Do contrário, a instrução é repetida até ele entendê-la. A tarefa é gravada e transcrita (de acordo com as normas do $\mathrm{NURC}^{1}$ ) para posterior análise.

Objetivando minimizar os efeitos de memória, as ilustrações ficam em frente ao participante ao longo do teste da tarefa. Não há tempo limite para produção e a única interferência do examinador, no decorrer da tarefa, é de encorajamento ao participante, caso ele não demonstre iniciativa na continuidade da história. $\mathrm{O}$ encorajamento é realizado através de falas como: "o que mais?", “o(a) senhor(a) pode continuar?", "uhum", "e depois, o que acontece?".

\section{Análise de dados}

As estratégias comunicativas foram analisadas de forma quanti/qualitativa intra e intergrupo (Quadro 1), a fim de ajudar a traçar o perfil dos grupos estudados. Para a análise qualitativa, foram investigadas as características e os tipos de estratégias utilizados. Para a análise quantitativa, foram somadas as quantidades totais de estratégias utilizadas na construção do texto oral pelos participantes.

Quadro 1. Estratégias comunicativas no texto oral

\begin{tabular}{|l|l|}
\hline \multicolumn{2}{|c|}{ (FÁVERO, ANDRADE E AQUINO, 2000; KOCH, 2008) } \\
\hline Hesitação & $\begin{array}{l}\text { Pausas curtas ou mais longas, alongamento das } \\
\text { palavras. }\end{array}$ \\
\hline Paráfrase & $\begin{array}{l}\text { Atividade de reformulação de um enunciado anterior, } \\
\text { mantendo uma relação de equivalência. }\end{array}$ \\
\hline Repetição & Repetição da última palavra ou frase enunciada \\
\hline Autocorreção & $\begin{array}{l}\text { Mudança de planejamento ao longo da construção } \\
\text { do texto oral }\end{array}$ \\
\hline Neologismo & Criação de uma palavra inexistente na língua. \\
\hline Parafasia & $\begin{array}{l}\text { Troca da palavra alvo por outra palavra semântica } \\
\text { ou fonologicamente relacionada }\end{array}$ \\
\hline Circunlóquio & $\begin{array}{l}\text { Dificuldade de discorrer sobre o tópico escolhido } \\
\text { (rodeios). }\end{array}$ \\
\hline
\end{tabular}

Fonte: Adaptado de Fávero, Andrade, Aquino (2000), Koch (2008).
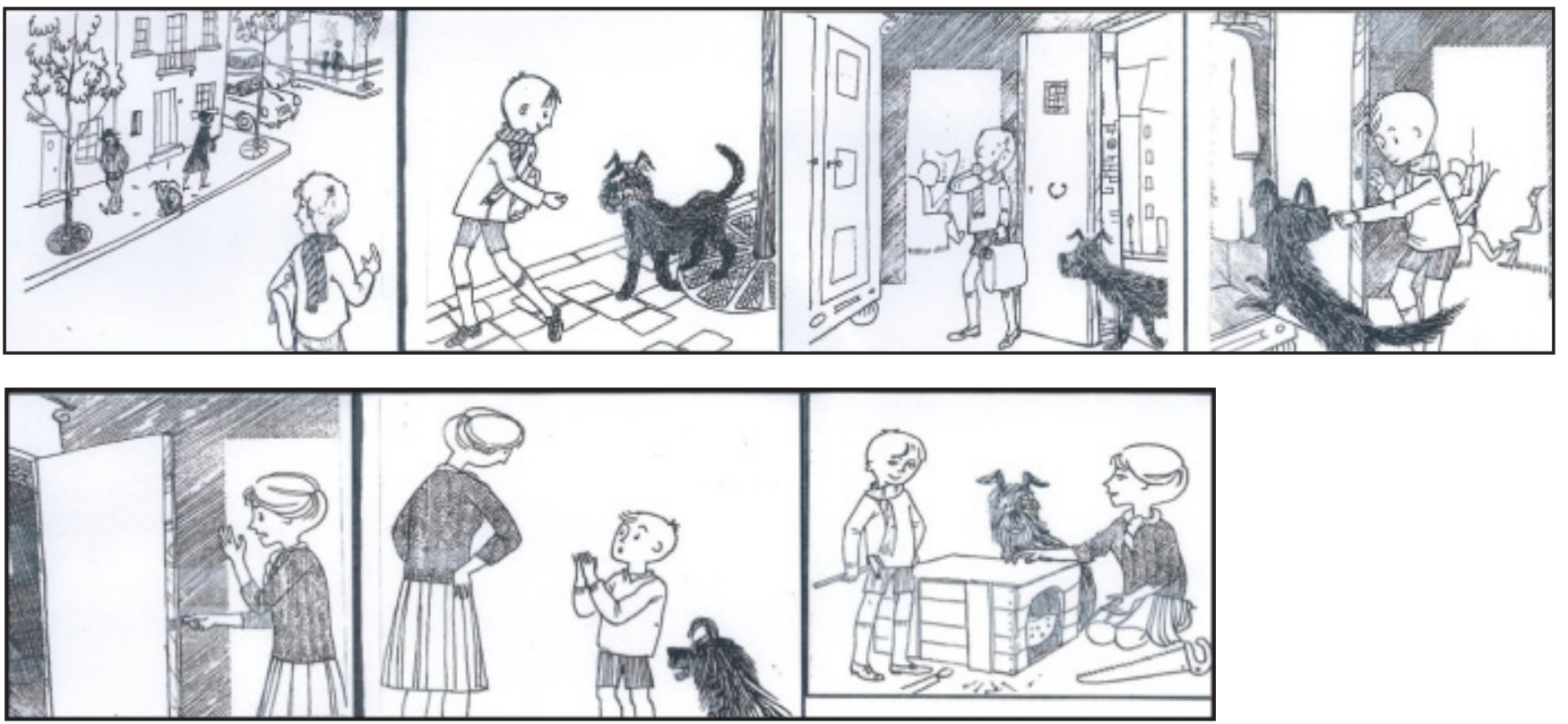

Figura 1. Instrumento linguístico de coleta de dados. Fonte: Le Bouef (1976).

\footnotetext{
1 Norma Urbana Culta. Norma utilizada para transcrição de entrevistas gravadas.
} 


\section{Resultados e discussão}

\section{Caracterização da amostra}

A amostra foi composta por 95 idosos.Os grupos clínicos foram diagnosticados com CCL amnéstico e DA em nível leve, denominados grupos estudo (GECCL e GEAD) em relação ao grupo controle (GC) - idosos saudáveis com baixa escolaridade. O GC também foi parâmetro para avaliar a variável escolaridade do grupo estudo formado por idosos saudáveis com alta escolaridade (GEAE) (Tabela 1).

Não houve diferença entre os grupos na idade, bem como na escolaridade, a qual diferiu apenas no grupo de idosos com alta escolaridade. No MEEM, os grupos GC, GECCL e GEDA tiveram diferença significativa entre si, indicando maior declínio no GEDA e GECCL, respectivamente. GEAE e GC não apresentaram diferença. $\mathrm{Na}$ escala GDS, os escores dos grupos não foram indicativos de depressão. O CDR foi 0,5 para GECCL e 1,0 para GEDA.

\section{Estratégias comunicativas - dados dos grupos clínicos}

Os resultados quantitativos obtidos na análise das ECs corroboraram parcialmente a hipótese de que GECCL e GEDA utilizam mais estratégias comunicativas compensatórias do que GC (DUOUNG et al., 2003). As ECs diferenciaram os grupos clínicos do GC, mas não os grupos com GECCL e GEDA entre si (Tabela 2).

GC, GECCL e GEDA produziram histórias semelhantes em número de palavras e proposições, mas diferiram na quantidade de ECs utilizadas, isto é, os grupos de idosos com GECCL e GEDA, mesmo com um número de palavras e proposições semelhantes ao do grupo $\mathrm{C}$, fizeram mais uso de ECs, mostrando uma diferença em termos de qualidade e não de quantidade. Esta diferença ilustra uma maior demanda cognitiva dos grupos GECCL e GEDA para a realização da tarefa.

As ECs são utilizadas na tentativa de suprir dificuldades discursivas encontradas. Embora seja perceptível que as ECs deixam o discurso mais truncado, no caso de ha-

Tabela 1. Caracterização da amostra

\begin{tabular}{|c|c|c|c|c|c|c|c|c|c|}
\hline & \multicolumn{2}{|r|}{$G^{A}$} & \multicolumn{2}{|c|}{ GECCLB } & \multicolumn{2}{|c|}{ GEDAC } & \multicolumn{2}{|c|}{ GEAED $^{\text {D }}$} & \multirow{2}{*}{ p } \\
\hline & $\mathbf{N}$ & M (DP) & $\mathbf{N}$ & $M(D P)$ & $\mathbf{N}$ & M (DP) & $\mathbf{N}$ & $M(D P)$ & \\
\hline Idade & 34 & $69,85(5,34)$ & 15 & $68,13(5,39)$ & 15 & $71,60(6,85)$ & 31 & $68,55(5,37)$ & $\begin{array}{l}0,333^{1} \\
0,265^{2}\end{array}$ \\
\hline Escolaridade & 34 & $4,94(1,85)$ & 15 & $3,87(1,35)$ & 15 & $4,27(1,75)$ & 31 & $15,42(2,15)$ & $\begin{array}{c}<0,001^{* 1} \\
0,115^{2}\end{array}$ \\
\hline MEEM & & $27,15(2,69)$ & & $22,40(4,06)$ & & $17,60(2,92)$ & & $28,26(1,96)$ & $\begin{array}{c}<0,001^{* 2} \\
<0,001^{* 3} \\
0,65^{3}\end{array}$ \\
\hline GDS & & $1,65(1,65)$ & & $3,20(2,24)$ & & $1,00(2,07)$ & & $1,61(1,66)$ & $\begin{array}{c}0,006^{\star 2} \\
0,006^{* 3} \\
0,515^{3} \\
0,934^{3}\end{array}$ \\
\hline CDR & & - & & 0,5 & & 1,0 & & - & \\
\hline
\end{tabular}

GEAE = grupo estudo com alta escolaridade; GC = grupo controle; GECCL = grupo estudo com Comprometimento Cognitivo Leve; GEDA = grupo estudo com doença de Alzheimer; MEEM = Mini Exame do Estado Mental; GDS = Escala de Depressão Geriátrica; CDR = Clinical Dementia Rating - Avaliação clínica de demência. *p $\leq 0,05$ indica diferença entre os grupos; ${ }^{1}$ Teste t Student; ${ }^{2}$ ANOVA; ${ }^{3}$ Análise post-hoc teste de Tukey HSD.

Tabela 2. Desempenho de GECCL e GEDA, em relação ao grupo GC, nas análises de estratégias comunicativas, número de palavras e número de proposições

\begin{tabular}{|c|c|c|c|c|c|c|c|c|}
\hline & \multicolumn{2}{|r|}{ GC } & \multicolumn{2}{|c|}{ GECCL } & \multicolumn{2}{|r|}{ GEDA } & \multirow{2}{*}{ Comparações } & \multirow{2}{*}{ p } \\
\hline & N & M (DP) & N & M (DP) & $\mathbf{N}$ & M (DP) & & \\
\hline Estratégias comuncativas & 34 & $13,09(5,58)$ & 15 & $23,93(13,76)$ & 15 & $28,47(9,27)$ & $\begin{array}{c}\mathrm{GC}<\mathrm{GECCL} \\
\mathrm{GC}<\mathrm{GEDA} \\
\mathrm{GECCL}<\mathrm{GEDA}\end{array}$ & $\begin{array}{c}<0,001^{* 1} \\
0,027^{* 2} \\
<0,001^{* 2} \\
0,119\end{array}$ \\
\hline Número palavras & 34 & $98,88(35,15)$ & 15 & $97,27(41,94)$ & 15 & $94,67(37,19)$ & $\begin{array}{c}\mathrm{GC}>\mathrm{GECCL} \\
\mathrm{GC}>\mathrm{GEDA} \\
\mathrm{GECCL}<\mathrm{GEDA}\end{array}$ & $0,935^{1}$ \\
\hline Número proposições & 34 & $16,56(6,29)$ & 15 & $17(8,62)$ & 15 & $16,60(6,35)$ & $\begin{array}{c}\mathrm{GC}<\mathrm{GECCL} \\
\mathrm{GC}>\mathrm{GEDA} \\
\mathrm{GECCL}>\mathrm{GEDA}\end{array}$ & $0,978^{1}$ \\
\hline
\end{tabular}

GEAE = grupo estudo com alta escolaridade; GC = grupo controle; GECCL = grupo estudo com Comprometimento Cognitivo Leve; GEDA = grupo estudo com doença de Alzheimer.

${ }^{*} \mathrm{p} \leq 0,05$ é considerado significante; ${ }^{1} \mathrm{ANOVA} ;{ }^{2}$ Análise post-hoc teste de Tukey HSD. 
ver um excesso de repetições, circunlóquios e paráfrases, elas também são importantes recursos cognitivos e comunicativos. Do ponto de vista pragmático, as ECs são vistas como um apoio à comunicação (DUONG et al., 2003; BRANDÃO; PARENTE; PEÑA-CASANOVA, 2010).

Em relação ao número de palavras, De Lira et al. (2014) encontraram diferenças entre o grupo controle e os dois grupos com DA (leve e moderada) na produção oral. Porém, tal diferença não foi encontrada entre os grupos com DA, que mostraram comprometimento nas unidades de informação. Assim, os grupos com DA evidenciaram prejuízos tanto qualitativos como quantitativos.

Cardebat, Démonet e Doyon (1993) não encontraram diferenças significativas entre o grupo com DA e controle na quantidade de palavras enunciadas, assim como nós. No entanto, por meio de outras análises linguísticas, como a análise das ECs, por exemplo, encontramos diferenças concernentes à qualidade das informações produzidas pelos grupos. Este resultado mostra que o número de palavras analisado isoladamente pode dizer pouco sobre a qualidade da produção e que ele, portanto, precisaria ser acrescido de outros tipos de análises. Parece não haver uma relação direta entre maior quantidade de palavras e/ ou proposições produzidas e qualidade discursiva.

No que tange ao CCL, Drummond et al. (2015) investigaram a quantidade de palavras na produção discursiva, por meio de instrumento pictórico, trazendo evidências que corroboram nossos achados. Assim como nós, os pesquisadores não encontraram diferenças entre o desempenho de idosos com CCL e controles, bem como entre idosos com CCL e DA. Tal resultado mostra que a quantidade de palavras não parece ser uma boa medida para diferenciar grupos clínicos com CCL e DA de idosos saudáveis, nem para diferenciar grupos clínicos (CCL e DA) entre si.

Quanto ao uso de ECs na produção discursiva, estes recursos podem dar pistas sobre a preservação da habilidade pragmática dos participantes, bem como do seu planejamento(BRANDÃO; PARENTE; PEÑACASANOVA, 2010). Nossos resultados mostraram que idosos com DA e CCL fizeram mais uso de ECs do que os idosos saudáveis, indicando, assim, uma preservação da habilidade pragmática no CCL e na DA. Ao mesmo tempo, o uso de ECs mostra uma maior dificuldade desses idosos na produção de um texto fluido, sem comentários pessoais, repetições, autocorreções e pausas.

Em oposição, De Lira (2014) e Cardebat, Démonet, Doyon (1993) evidenciaram dificuldades pragmáticas presentes na DA, as quais estão relacionadas aos modalizadores enunciativos. Conforme Cardebat, Démonet, Doyon (1993), os modalizadores se apresentam como avaliações feitas pelo sujeito sobre o conteúdo da história e demonstram uma preocupação consciente sobre suas dificuldades na produção realizada. Prejuízos desta ordem estiveram presentes nas produções do grupo com DA e controle dos estudos citados, mas não os diferenciaram. No presente estudo, GEDA se destacou na utilização de estratégias gerais, em comparação ao $\mathrm{GC}$, mostrando uma preservação da habilidade pragmática. $\mathrm{O}$ maior uso de estratégias dos grupos com CCL e DA pode estar associado a dificuldades no planejamento, isto é, na formulação do texto. Estas falhas no processamento podem ocorrer e precisam ser resolvidas. A iniciativa de resolução do problema mostra a capacidade do sujeito em adequar-se ao contexto (VAN DIJK, 2003).

$\mathrm{Na}$ análise quali-quantitativa dos tipos de ECs, as estratégias menos utilizadas por todos os grupos foram neologismo e parafasia. GECCL e GEDA fizeram mais uso das estratégias hesitação e circunlóquio. Nos grupos clínicos a estratégia de repetição apareceu em menor número do que as estratégias hesitação e circunlóquio. A EC de repetição diferenciou o grupo GEDA de GC, mas não GECCL e GC. Já as ECs hesitação e circunlóquio diferenciaram os grupos GEDA e GC, bem como GECCL e GC (Tabela 3).

Tabela 3. Desempenho de GECCL e GEDA, em relação ao GC, na análise quali-quantitativa das estratégias comunicativas

\begin{tabular}{|c|c|c|c|c|c|c|c|}
\hline \multicolumn{8}{|c|}{ Estratégias Comuncativas - História do Cachorrinho } \\
\hline & \multicolumn{2}{|c|}{ GC $(n=34)$} & \multicolumn{2}{|c|}{ GECCL $(n=15)$} & \multicolumn{2}{|c|}{ GEDA ( $n=15)$} & \multirow{2}{*}{ p Comparações } \\
\hline & soma & média & soma & média & soma & média & \\
\hline Hesitação* & 336 & 9,58 & 224 & 14,63 & 258 & 17,2 & $\begin{array}{l}0,001^{*} \text { GEDA>GC } \\
0,022^{*} \text { GECCL>GC }\end{array}$ \\
\hline Paráfrase & 1 & 0,02 & 5 & 0,03 & 8 & 0,53 & \\
\hline Repetição* & 31 & 0,91 & 29 & 1,93 & 33 & 2,2 & $0,012^{*} \mathrm{GEDA}>\mathrm{GC}$ \\
\hline Autocorreção & 14 & 0,41 & 13 & 0,98 & 3 & 0,2 & \\
\hline Neologismo & 1 & 0,02 & 3 & 0,2 & 0 & 0,0 & \\
\hline Parafasia & 2 & 0,05 & 1 & 0,06 & 0 & 0,0 & \\
\hline Circunlóquio* & 71 & 2,08 & 83 & 5,53 & 124 & 8,26 & $\begin{aligned}<0,001^{*} & \text { GEDA }>G C \\
0,004^{*} & \text { GECCL }>G C\end{aligned}$ \\
\hline Total estratégias grupo & 445 & 13,08 & 359 & 23,93 & 427 & 28,46 & \\
\hline
\end{tabular}

GEAE = grupo estudo com alta escolaridade; GC = grupo controle; GECCL = grupo estudo com Comprometimento Cognitivo Leve; GEDA = grupo estudo com doença de Alzheimer.

*ANOVA - Análise post-hoc teste de Tukey HSD. 
A maioria dos estudos que investigaram as ECs na DA e no CCL verificaram o desempenho dos grupos com relação à estratégia de repetição (DUONG et al., 2003; DIJKSTRA et al., 2002, 2004). Há diferenças entre o desempenho de idosos com DA e controles na repetição de palavras (DUOUNG et al., 2003; DIJKSTRA et al., 2002, 2004; FRASER; MELTZER; RUDZIXCZ, 2016; YANCHETA, 2016),corroborando, assim, nossos achados. Cabe ressaltar que a repetição é uma característica do discurso oral, podendo exercer diferentes funções como, por exemplo, servir de recurso retórico, o qual produz efeitos semânticos de persuasão; ou ser utilizada como um recurso coesivo de referenciação, que marca passagens novas e retomadas $(\mathrm{KOCH}, 2008)$. É importante também que se identifique se as repetições estão no tópico ou não (BRANDÃO et al., 2009). Como EC a repetição exerce função de manutenção do turno e de ganho de tempo para o planejamento (KOCH, 2008). A repetição pode ser uma estratégia para compensar dificuldades(TOMOEDA; BAYLES, 1993). As repetições foram seguidas de muitas hesitações, o que enfatiza a necessidade de mais tempo para o processamento das informações, deste modo, reforçando seu uso como ECs. No quesito repetição, o grupo com GECCL se assemelhou ao GC do que com GEDA, não se diferenciando dele. No desempenho linguístico, GECCL ora se parece com GC ora com GEDA (DRUMMOND et al., 2015; ALBERT et al., 2011). Abaixo, segue o fragmento de um texto do GECCL, o qual traz trechos de repetições:

\footnotetext{
"tem um menino... olhou pro lado...ia na rua... olhou pro lado... tinha um cachorro parado... e... sozinho.... pessoas passando... passando... (...) o cachorrinho acompanhou... acompanhou ele até na casa dele... ele entrou (...)"
}

Hesitação foi outro tipo de EC que diferenciou os grupos, sendo a EC mais frequente. Neste caso, o GECCL se pareceu mais com o GEDA do que com o GC. Circunlóquio foi o segundo tipo de EC mais utilizado, diferenciando os grupos clínicos do GC. O circunlóquio ocorre quando há uma evidente dificuldade em discorrer sobre o tópico escolhido (ORTIZ, 2010). No caso dos idosos com GEDA, eles tangenciaram o tema, mas, ainda assim, tentaram estabelecer o contato com o interlocutor. A primeira etapa do planejamento de produção textual é o planejamento pragmático (BRANDÃO, 2006). $K$-device seria o responsável pela busca de informações, o qual monitoraria e limitaria as informações necessárias à produção do texto (VAN DIJK, 2003). Na fase de planejamento pragmático, a pessoa tenta articular a construção do texto com as informações disponíveis na memória, bem como adequá-las à coerência do texto. Como a articulação das informações semânticas pode estar prejudicada na DA, o indivíduo pode discorrer, por exemplo, sobre algum tema relacionado ou fazer rodeios para manter a interação, demonstrando um $K$-device em monitoramento. O GEDA inseriu diversos trechos ao longo dos textos, fazendo rodeios. Alguns trechos narrativos de experiência pessoal também apareceram nas produções das pessoas com DA, conforme mostra o exemplo, abaixo:

"quando eu era criança assim também morava ali no Passo da Areia... tinha os cachorrinhos também quando era criança tudo... e eu trabalhava bastante... lavrava o campo tudo... eles tão caminhando na calçada da rua né... é aqueles cachorrinho na calçada... o poste aqui... aqui ta dentro de casa... o cachorrinho... aqui ta conversando com o cachorrinho... o gurizinho... (...)"

No exemplo citado, há o início de uma narrativa pessoal, quando o participante deve ter associado a figura do cachorro a uma experiência remota, demonstrando uma memória autobiográfica preservada, típico de pessoas com DA em fase inicial (BRANDÃO; PARENTE; PEÑACASANOVA, 2010). Na sequência, o participante parece retornar ao estímulo das figuras, em uma tentativa de estabelecer o cenário da história e narrar os acontecimentos. Porém, a narração da história não se efetiva, visto que o participante insere diversas descrições, as quais predominam. A dificuldade de o participante iniciar a narração da história solicitada, bem como a inserção do trecho da narrativa pessoal, mostram que, na falta de recursos, o participante utilizou uma estratégia comunicativa de algo que era familiar para ele, a fim de responder à solicitação, satisfazendo o nível pragmático. Esta ocorrência vem ao encontro do modelo de Van Dijk (2003), mostrando que o $k$-device monitora o manejo do conhecimento e a adequação pragmática. É importante ressaltar que o circunlóquio, por envolver esforço cognitivo na resolução de uma tarefa, pode ser considerado uma importante EC, do ponto de vista pragmático (DUONG et al., 2003). Entretanto, se observado pelo prisma semântico e de coerência do texto, o circunlóquio não apresentará o mesmo efeito positivo.

A hesitação, o circunlóquio e a repetição conferem aos indivíduos maior tempo para planejar a fala. Todos os grupos com baixa escolaridade fizeram uso destes recursos. Embora GECCL e GEDA tenham feito mais uso das ECs de repetição e hesitação do que GC, GECCL não apresentou diferença significativa em relação ao $\mathrm{GC}$ na EC de repetição. Já o GEDA apresentou diferença significativa para ambas as estratégias.

As ECs desenvolvem-se ao longo do desenvolvimento da linguagem, podendo automatizarem-se e fazerem parte do conhecimento implícito. Porém, algumas delas parecem estar ligadas à preservação da habilidade de monitoramento da produção discursiva, compondo o conhecimento explícito do sujeito (BRANDÃO; PARENTE; PEÑA-CASANOVA, 2010). 
A análise de ECs mostrou que GECCL e GEDA possuem a habilidade pragmática preservada no que se refere a este recurso, especialmente no caso de a produção oral ser realizada com apoio de pistas visuais. As ECs circunlóquio e hesitação, portanto, são importantes marcadores de CCL e DA. No que tange ao desempenho cognitivo e linguístico, essa abordagem altera a visão tradicional, a qual tem por objetivo investigar o déficit por si só (BRANDÃO; PARENTE; PEÑA-CASANOVA, 2010).

\section{Estratégias comunicativas - dados dos grupos saudáveis variável escolaridade}

GEAE diferenciou-se de GC em todos os três aspectos (Tabela 4). O grupo GEAE utilizou mais palavras e proposições e fez menos uso de ECs para a produção da narrativa oral do que GC.

Juncos-Rabadán, Pereiro e Rodríguez (2005), ao relacionar idade e escolaridade na produção de narrativas baseadas em figuras, verificaram que as narrativas dos idosos foram mais longas do que a dos adultos jovens e com menor conteúdo informativo. Entretanto, no caso dos idosos com maior escolaridade, os textos foram mais longos, mas com maior qualidade do conteúdo e clareza de referentes.

O impacto positivo da variável escolaridade, verificado no estudo de Juncos-Rabadán, Pereiro e Rodríguez (2005), foi igualmente observado no presente estudo, corroborando, portanto, a hipótese de que a escolaridade exerce influência no desempenho linguístico dos idosos saudáveis.

Os textos dos idosos do GEAE foram mais longos, porém com maior densidade informacional e menor uso de estratégias. Este resultado corrobora a importância da escolaridade na cognição, ancorando a Hipótese de Reserva Cognitiva (HRC) no envelhecimento (WHALLEY et al., 2004). A utilização de ECs pelo GEAE era menos esperada do que pelos grupos com DA e CCL, por exemplo, que necessitavam fazer mais compensações. GEAE potencialmente tem suas funções cognitivas mais preservadas e espera-se igualmente que a habilidade pragmática de comunicação esteja intacta.

Em um estudo com reconto de textos, Parente e colegas (1999) verificaram que os idosos usam mais ECs, quando a memória de trabalho se mostra prejudicada. Outros estudos envolvendo idosos mostraram que a variável escolaridade foi a que mais contribuiu para explicar os dados (MORAES et al., 2012).

$\mathrm{Na}$ análise quali-quantitativa, as estratégias neologismo e parafasia não foram utilizadas pelo GEAE, conforme esperado, pois estas estratégias eram mais prováveis de aparecerem nas narrativas dos grupos clínicos, que possuem maiores dificuldades com o acesso lexical. Houve poucas ocorrências de paráfrase e autocorreção no GEAE. As ECs menos utilizadas pelo GC foram paráfrase, neologismo e parafasia. Já as ECs mais utilizadas por ambos os grupos foram hesitações, repetições e circunlóquios (Tabela 5).

Tabela 4. Desempenho do grupo com GEAE, em relação GC, nas análises das estratégias comunicativas, número de palavras e proposições

\begin{tabular}{|c|c|c|c|c|c|c|}
\hline & \multicolumn{2}{|r|}{ GEAE } & \multicolumn{2}{|r|}{ GC } & \multirow{2}{*}{ Comparações } & \multirow{2}{*}{ p } \\
\hline & $\mathbf{N}$ & M\% (DP) & N & M \% (DP) & & \\
\hline Estratégias Comuncativas & 31 & $10,19(4,50)$ & 34 & $13,09(5,58)$ & GEAE $<$ GC & $0,026^{* 1}$ \\
\hline Número Palavras & 31 & $125,29(46,21)$ & 34 & $98,88(35,15)$ & GEAE >GC & $0,011^{* 1}$ \\
\hline Número Proposições & 31 & $21,35(8,27)$ & 34 & $16,56(6,29)$ & GEAE >GC & $0,010^{*}$ \\
\hline
\end{tabular}

GEAE = alta escolaridade; $\mathbf{G C}=$ comparativo de baixa escolaridade

${ }^{*} \mathrm{p} \leq 0,05$ é considerado significante; ${ }^{1}$ Teste t Student.

Tabela 5. Desempenho do grupo com GEAE, em relação ao GC, na análise quali-quantitativa das estratégias comunicativas

\begin{tabular}{|c|c|c|c|c|c|c|}
\hline \multicolumn{7}{|c|}{ Estratégias Comuncativas - História do Cachorrinho } \\
\hline & \multicolumn{2}{|c|}{ GEAE $(n=31)$} & \multicolumn{2}{|c|}{ GC $(n=34)$} & \multirow{2}{*}{$\mathbf{p}$} & \multirow{2}{*}{ Comparações } \\
\hline & soma & média & soma & média & & \\
\hline Hesitação & 256 & 8,12 & 336 & 9,58 & 0,182 & $C>G E A E$ \\
\hline Paráfrase & 6 & 0,19 & 1 & 0,02 & & \\
\hline Repetição & 11 & 0,35 & 31 & 0,91 & $0,008^{*}$ & $C>G E A E$ \\
\hline Autocorreção & 8 & 0,25 & 14 & 0,41 & & \\
\hline Neologismo & 0 & 0,0 & 1 & 0,02 & & \\
\hline Parafasia & 0 & 0,0 & 2 & 0,05 & & \\
\hline Circunlóquio & 39 & 1,25 & 71 & 2,08 & $0,030^{*}$ & $\mathrm{C}>\mathrm{GEAE}$ \\
\hline Total de estratégias por grupo & 316 & 10,19 & 445 & 13,08 & & \\
\hline
\end{tabular}

GEAE = alta escolaridade; GC = comparativo de baixa escolaridade

*Teste t Student. 
Das estratégias mais frequentes, repetição e circunlóquio diferenciaram os grupos, ao passo que hesitação não. A hesitação marca uma quebra na linearização, na formulação da ideia. O objetivo é ganhar tempo para encontrar a palavra desejada. Estudos mostram que idosos podem ser mais lentos na resolução de tarefas e demandar de um maior tempo para realizá-las com eficiência, tendo desempenho semelhante ao de jovens adultos (PERGHER; STEIN, 2003). Esta necessidade de maior tempo dos idosos na resolução de tarefas pode explicar a frequência de hesitações no discurso de idosos tanto de baixa como de alta escolaridade. Neste quesito a escolaridade não mostrou diferença entre os grupos. Entretanto, se avaliado o desempenho global dos idosos com alta escolaridade na frequência de uso de ECs, é possível verificar o fator protetor da escolaridade, pois o GEAE fez marginalmente menor uso de ECs.

Os resultados da análise quali-quantitativa das ECs mostram que a EC hesitação não é utilizada apenas por idosos saudáveis com baixa escolaridade, mas igualmente por idosos com alta escolaridade, sendo de uso comum no envelhecimento saudável. No entanto, o GEAE a utilizam em menor número. Já as ECs de repetição e circunlóquio são capazes de diferenciar os idosos pela escolaridade, mostrando o fator protetivo da HRC. As ECs de repetição e circunlóquio parecem ser, assim, importantes marcadores de reserva cognitiva e compensações de dificuldades cognitivas no envelhecimento saudável, sendo relevantes na diferenciação de idosos saudáveis com alta e baixa escolaridade.

\section{Considerações finais}

Os grupos de estudo com CCL e DA fizeram uso de ECs. A análise quanti-qualitativa das ECs revelou diferença entre os grupos com CCL, DA e o grupo controle nos itens hesitação, repetição e circunlóquio. Apenas os itens hesitação e circunlóquio diferenciaram o grupo estudo com CCL do grupo controle.

A presença de estratégias comunicativas na produção oral dos idosos com CCL e DA, do ponto de vista pragmático, trouxe pistas sobre a preservação da habilidade pragmática destes idosos, uma vez que eles mantiveram a comunicação ativa com o interlocutor, mesmo apresentando dificuldades na realização das tarefas. As estratégias comunicativas mostraram-se um apoio à comunicação na $\mathrm{DA}$ e no $\mathrm{CCL}$, especialmente a hesitação e o circunlóquio. Tal adequação pragmática foi associada com o componente K-Device, o qual tem por objetivo adequar a fala do sujeito ao contexto. O conhecimento de estratégias compensatórias na comunicação, especialmente por parte de indivíduos com acometimento neurológico, é de extrema relevância para cuidadores, familiares e demais profissionais da área da saúde.

Quanto ao impacto da variável escolaridade na produção de narrativa oral de idosos saudáveis, as evidências encontradas endossaram sua importância, vindo ao encontro da Hipótese de Reserva Cognitiva.

\section{Referências}

ALBERT, Marilyn S. et al. The diagnosis of mild cognitive impairment due to Alzheimer'sdisease: Recomendations from the National Institute on Aging-Alzheimer's Association workgroups on diagnostic guideline for Alzheimer's disease. Alzheimer's \& Dementia, v. 7, p. 270-279, 2011.

BRANDÃO, Lenisa. Perfil discursivo de pessoas com Doença de Alzheimer. 2005. 185 f. Tese (Doutorado) - Faculdade de Psicologia, Universidade do Rio Grande do Sul, Porto Alegre, 2005.

BRANDÃO, Lenisa. Produção da linguagem e envelhecimento. In: PARENTE, M.A.M.P. e Col. Cognição e envelhecimento. Porto Alegre: Artmed, 2006. p. 133-152.

BRANDÃO, Lenisa et al. Cognition and discourse production in Alzheimer's disease: using informative prompts. Psychology \& Neuroscience, v. 2, n. 2, p. 147-155, 2009.

BRANDÃO, L.; PARENTE, M. A. M. P.; PEÑA-CASANOVA, J. Estratégias comunicativas de pessoas com Alzheimer. Psicologia: reflexão e crítica, v. 23, n. 2, p. 308-316, 2010.

CARDEBAT, Dominique; DEMONET, Jean-François; DOYON, Bernard.Narrative discourse in dementia. In: BROWNELL, H. H; YVES, Joanette (Ed.). Narrative discourse in neurologically impaired and normal aging adults. San Diego: Singular; 1993. p. 317-22.

CAIXETA, Leonardo; PINTO, Henrique; SOARES, Vânia Lúcia; SOARES, Cândida. In: CAIXETA, Leonardo; TEIXEIRA, Antônio (Org.). Neuropsicologia Geriátrica: neuropsiquiatria cognitive em idosos. Porto Alegre: Artmed, 2014.

De LIRA, Juliana. Análise dos aspectos macrolinguísticos e desenvolvimento de um índice de avaliação do discurso oral em pacientes com doença de Alzheimer. 2014. $138 \mathrm{f}$. Tese (Doutorado, não publicada) - Faculdade de Medicina, Universidade Federal de São Paulo, São Paulo, 2014.

DIJKSTRA, Katinka et al.My recaller is on vacation: Discourse analysis of nursing-home residents with dementia. Discourse Processes, v. 33, n. 1, p. 53-76, 2002.

DIJKSTRA, Katinka et al.Conversational coherence: discourse analysis of older adults with and without dementia. Journal of Neurolinguistics, v. 17, p. 263-283, 2004.

DUONG, Anh; TARDIF, Andreanne; SKA, Bernadette. Discourse about discourse: What is it and how does it progress in Alzheimer's disease? Brain and Cognition, v. 53, p. 177-180, 2003.

DRUMMOND, Cláudia.; COUTINHO, Gabriel.; FONSECA, Rochele et al. Deficits in narrative discourse elicited by visual stimuli are already presented in patients with mild cognitive impairment. Frontiers in Aging Neuroscience, v. 7, p. 1-11, 2015 . 
FÁVERO, Leonor Lopes; ANDRADA, Maria Lúcia C. V. O.; AQUINO, Zilda G. Oralidade e escrita: perspectivas para o ensino de língua materna. São Paulo: Cortez, 2000.

FICHMAN, Helenice Charchat; OLIVEIRA, Rosinda Martins; FERNANDES, Conceição Santos.Neuropsychological and neurobiological markers of the preclinical stage of Alzheimer's disease. Psychology \& Neuroscience, v. 4, n. 2, p. 245-253, 2011.

FRASER, Kathleen; MELTZER, Jed; RUDZICZ, Frank. Linguistic features identify Alzheimer's disease in narrative speech. Journal of Alzheimer's Disease, v. 49, n. 2, p. 407-422, 2016.

GOMES, Irênio; TERRA, Newton. Doença de Alzheimer. In: IZQUIERDO, Ivan et al. (Org.). Envelhecimento, memória e doença de Alzheimer. Porto Alegre: EdiPUCRS, 2015. p. 29-64.

JAKUBOVICZ, Regina; CUPELLO, Regina. Introdução à afasia: elementos para o diagnóstico e terapia. Rio de Janeiro: Revinter, 2005.

JERÔNIMO, Gislaine Machado; HÜBNER, Lilian Cristine. Produção discursiva e comunicação na doença de Alzheimer uma revisão de pesquisas. Revista Neuropsicologia Latinoamericana, v. 6, n 3, p. 4-15, 2014.

JUNCOS-RABADÁN, Onésimo; PEREIRO, Arturo X.; RODRÍGUEZ, María Soledad. Narrative speech in aging: quantity, information content, and cohesion. Brain and Language, v. 95, p. 423-434, 2005.

$\mathrm{KOCH}$, Ingedore. O texto e a construção dos sentidos. São Paulo: Contexto, 2008.

LE BOUEF, Christine. Raconte - 55 historiettes en images. Paris: L'Ecole, 1976.

MCKHANN, Guy et al. The diagnosis of dementia due to Alzheimer's disease: Recommendations from the National Institute on Aging-Alzheimer's Associations workgroups on diagnostic guidelines for Alzheimer's disease. Alzheimer's \& Dementia, v. 7, p. 263-269, 2011.
ORGANIZAÇÃO MUNDIAL DE SAÚDE - OMS. Disponível em: <http://sbgg.org.br/oms-divulga-relatorio-sobreenvelhecimento-e-saude/>. Acesso em: 02 jun. 2017.

ORTIZ, Karin Zazo. Terapia nos distúrbios compreensivos. In: ORTIZ, Karin Zazo (Org.). Distúrbios neurológicos adquiridos: linguagem e cognição. São Paulo: Manole, 2010. p. 110-135.

PARENTE, Maria Alice; CAPUANO, Andréa; NESPOULOUS, Jean Luc. Ativação de modelos mentais no recontar de histórias por idosos. Psicologia: reflexão e crítica, v. 12, n. 1, 1999.

SCHWARTZ, Bennett; METCALFE, Janet. Tip-of-the-tongue (TOT) states: retrieval, behavior, and experience. Memory \& Cognition, v. 39, p. 737-749, 2011.

TOLEDO, Cíntia Matsuda et al. Analysis of macrolinguistic aspects of narratives from individuals with Alzheimer's disease, mild cognitive impairment, and no cognitive impairment. Alzheimer's \& Dementia: Diagnosis, Assessment \& Disease Monitoring, 2017.

TOMOEDA, Cheryl; BAYLES, Kathryn. Longitudinal effects of Alzheimer Disease on discourse production. Alzheimer Disease and Associated Disorders, v. 7, n. 4, p. 223-236, 1993.

TUBERO, Ana. Paula. Parafasia: o quiproquó das palavras. In: MORATO, Edwiges. (Org.). A semiologia das afasias: perspectivas linguísticas. São Paulo: Cortez, 2010. p. 62-101.

WEIRATHER, Randy. Communication strategies to assist comprehension in dementia. Hawal'l Medical Journal, v. 69, p. 72-79, 2010.

YANCHEVA, Maria. Automatic assessment of information content in speech for detection of dementia of the Alzheimer type. 2016. $70 \mathrm{f}$. Tese (Doutorado) - University of Toronto, Canadá, 2016.

Recebido: 30/09/2017

Aprovado: 10/02/2018

Contato:

Gislaine Machado Jerônimo < gislaine.mjeronimo@gmail.com> 